\title{
Quantification of urinary chorionic gonadotropin in spontaneous abortion of pre-clinically recognized pregnancy: Method development and analytical validation
}

\author{
M. Fátima Reis ${ }^{\mathrm{a}, *}$, Pedro Aniceto ${ }^{\mathrm{a}}$, Pedro Aguiar ${ }^{\mathrm{b}}$, Filipa Simão ${ }^{\mathrm{a}}$, Susana Segurado ${ }^{\mathrm{a}}$ \\ anstitute of Preventive Medicine, Faculty of Medicine, University of Lisbon, Av. Prof. Egas Moniz, Edificio Egas Moniz, Piso 0, \\ Ala C, 1649-028 Lisbon, Portugal \\ ${ }^{\mathrm{b}}$ National School of Public Health, New University of Lisbon, Lisbon, Portugal
}

\begin{abstract}
Determination of environmental impacts on reproductive health and specifically on the incidence of early spontaneous abortion requires accurate estimates of the latter. This negative reproductive outcome can be detected by the pattern of elevation and decline of human chorionic gonadotropin (hCG) levels near and shortly beyond the expected time of implantation, requiring daily biomonitoring of hCG levels during the relevant period of the menstrual cycle. Prospective pregnancy studies to assess effects of potentially toxic exposures on human reproductive outcomes can involve up to three menstrual cycles and a huge number of samples in each, for the quantification of the inherently very low hCG levels usually can be determined only in serum. The invasive nature of blood collection, the number of samples needed for the development of prospective studies, and the lack of quantitative methods for the determination of low hCG levels in urine point to the need for collecting urine rather than blood and make it imperative to develop suitable quantitative methods for biomonitoring of very low levels of hCG in urine. This paper describes the development and validation procedures of an automated solid-phase two-site chemiluminescent immunometric assay for the quantification of urinary hCG in early pregnancy and early pregnancy loss. For the validation, both undiluted and diluted urine and control samples have been prepared. From the results, it can be concluded that the assay has a calibration range that extends to $5000 \mathrm{mIU} / \mathrm{ml}$, with a detection limit of approximately $1.2 \mathrm{mIU} / \mathrm{ml}$, practically identical to that found by the IMMULITE 2000 manufacturer's validation study. The intra- and inter-assay precision ranges up to a maximum of around 7\%, meaning that the practical limit for functional sensitivity can be established as low as $10 \%$. This means that the immunoassay from DPC ${ }^{\mathbb{R}}$ can identify, with relatively high confidence, non-pregnant women and the typical "rise and fall" pattern of early pregnancy loss through analysis of urine samples. Results also lead to the conclusion that there is a very good agreement between expected and observed urinary hCG levels indicative of good immunoassay accuracy for the studied range of hCG concentrations. In terms of analyte stability, it can be concluded that urinary hCG is stable under the expected conditions required for ongoing investigations that include temperatures of $2-8{ }^{\circ} \mathrm{C}$ for up to $48 \mathrm{~h}$ and temperatures of around $-20^{\circ} \mathrm{C}$ for longer periods that can extend to over 3 months.
\end{abstract}

(C) 2007 Elsevier GmbH. All rights reserved.

Keywords: hCG biomonitoring; Urine; Quantitative immunoassay; DPC ${ }^{\circledR}$ IMMULITE 2000; Validation

\footnotetext{
*Corresponding author. Tel.: +35121 7985100; fax: +351217999487

E-mail address: mfreis@fm.ul.pt (M. Fátima Reis).
} 


\section{Introduction}

Environmental impacts on reproductive health have become a pressing issue at a time when birth rates are widely falling in developed countries. Several pollutants have been associated with a variety of adverse reproductive outcomes (Bhatt, 2000; Shahara et al., 1998) and, contrary to traditional thinking that only in utero exposures are of concern, growing scientific evidence shows the potentially additional importance of parental exposures during critical pre- and periconceptional windows (Chapin et al., 2004). A spectrum of negative reproductive outcomes can be conceptualized for assessing reproductive toxicants. Furthermore, given the ethical limitations of experimental study designs in spite of their strength, the choice is among observational designs for epidemiological investigations on the effects of potentially toxic exposures on human reproductive end points. Preferable to retrospective studies, prospective cohort designs are of election, mainly due to the possibility of ensuring not only temporal ordering between exposure(s) and outcome(s), but better performance in the measurement of exposure as well as of relevant co-variates, and minimum potential information bias (Buck et al., 2004). Focussing investigation on pregnant women does not allow the ascertainment of exposures at critical peri-conceptional windows, or the assessment of early reproductive outcomes, in particular spontaneous abortion before clinically recognized pregnancy. Suspicions that exposure to certain pollutants (e.g., heavy metals and dioxins) may be linked to spontaneous abortion are aggravated by a generalized inaccuracy in calculated incidence rates, as the loss of a pregnancy before it is clinically recognizable often leads to its never being accounted for as such. A prospective pregnancy study with pre-pregnancy enrolment will allow for the longitudinal assessment of exposures, only in women or in both parents, beginning prior to pregnancy and continuing throughout and beyond it (Buck et al., 2004). Since the ultimate objective is to relate chemical exposures to adverse health effects, human biomonitoring should be used. This type of study will also allow a wide range of reproductive outcomes to be assessed, namely that of spontaneous abortion before pregnancy becomes clinically evident (Tingen et al., 2004), providing that both instances of very early pregnancy and subsequent abortion can be accurately detected and documented.

Human chorionic gonadotropin (hCG) is a glycoprotein hormone present in serum and urine during pregnancy whose no covalently associated subunits ( $\alpha$ and $\beta$ subunits) can be detected as early as 6 days after conception (Vankrieken et al., 2000), making it a prime candidate for pre-empting pregnancy detection.
Basal hCG levels in non-pregnant healthy women can be lower than $5 \mathrm{mIU} / \mathrm{ml}$. After conception and during the first trimester of pregnancy, circulating levels rise exponentially, doubling every $48 \mathrm{~h}$, to a peak on the order of $50000-100000 \mathrm{mIU} / \mathrm{ml}$ or higher, at about 10 weeks of pregnancy. Throughout the first part of the second trimester concentrations of hCG decrease to approximately one-fifth of the maximum by the 16th week of gestation, and remain in this steady level until term (Cole, 1997; Vankrieken et al., 2000). After delivery or in case of abortion, hCG decreases rapidly (Wilcox et al., 1988). Therefore, the pattern of elevation and decline in hCG levels can be used to detect spontaneous abortion. However, in the case of early pregnancy loss ( $\leqslant 3$ weeks of gestation), concentrations of hCG are still very low and the difference between hCG levels distinguishing pregnancy from pregnancy loss can be very small, requiring highly sensitive quantitative methods.

To detect early spontaneous abortion, the patterns of elevation and decline of hCG levels have to be obtained through daily biomonitoring of hCG levels during the relevant period of the menstrual cycle. Although there is a wide range of qualitative tests available for determining whether a urine sample has an hCG concentration above or below a cut-off value (that is usually defined as $30 \mathrm{mIU} / \mathrm{ml}$ ), determination of lower levels of hCG relay on analyte quantification in serum samples. However, daily collection for several (up to three) menstrual cycles and for at least 2 weeks in each regular cycle, and possibly longer in the case of non-regular menstrual cycles makes blood sampling impractical (and unethical). This points to the need for collecting daily urine samples rather than using much more invasive blood collection and makes it imperative to develop suitable quantitative methods for the biomonitoring of very low levels of hCG in urine. Blood collection is also needed for the assessment of chemical exposures using human biomonitoring.

When a new or modified analytical method is considered for use in routine analysis of a large number of samples, it has to be validated in order to demonstrate that the method is appropriate for the analysis of the matrices and levels of concentration to which it will be applied. Therefore, the aim of the current study is to develop and validate a suitable method for the quantification of the very low levels of urinary hCG commonly found in early pregnancy and in early pregnancy loss, starting from an existing automated solid-phase, two-site, chemiluminescent enzyme immunometric assay (IMMULITE $2000 \mathrm{hCG}$ immunoassay from Diagnostic Products Corporation $\mathrm{DPC}^{\mathbb{R}}$ ) formerly intended for use in the quantitative measurement of hCG in serum and for qualitative determinations of urinary $\mathrm{hCG}$, as an aid to the detection of clinically recognized pregnancy (Cole 
et al., 2001; DPC ${ }^{\circledR}$ (Diagnostic Products Corporation), 2005). For validation, the performance characteristics of the immunoassay for hCG in urine are determined and demonstrated. The methods for establishing these characteristics are mainly based on guidelines provided by the Food and Drug Administration of the United States (FDA (Food and Drug Administration), 2001), although several other documents from the scientific literature have also been used (BDS (BioDetection Systems), 2003; Sackrison et al., 2002; Findlay et al., 2000; Shah et al., 2000; NCCLS (National Committee for Clinical Laboratory Standards), 1986, 1999; Reis et al., 1994).

\section{Materials and methods}

\section{Equipment and reagents}

Since the immunoassay has to be used for the quantification of very low hCG levels in urine, adjustments have been performed by the manufacturer's representative in the software reporting range of an existing IMMULITE 2000 Analyzer - DPC ${ }^{\circledR}$ (Spencer, 1989), in order to allow quantification of urinary hCG below $30 \mathrm{mIU} / \mathrm{ml}$, the previously defined clinical "cutoff value" (DPC ${ }^{\circledR}$ (Diagnostic Products Corporation), 2005; Trullols et al., 2005).

Validation of the automatic immunoassay used the following reagents: chemiluminescent substrate module LUMIGEN $^{\circledR}$ PPD; probe wash module concentrate; hCG reagent wedge (bovine alkaline phosphatase and sheep polyclonal antibody); hCG bead pack (beads coated with monoclonal murine anti-hCG); hCG sample diluents (hCG-free human serum); multivalent control module immunoassay, with tri-level quality control samples $\left(\mathrm{CON}^{\mathrm{R}}{ }^{\mathrm{R}}\right.$ ); and $\mathrm{hCG}$ adjustors (two vials low and high levels - of lyophilized hCG in a hCG-free human serum matrix, with preservative). Purified deionized water was prepared using an ELIX $^{\circledR} 3$ ZLXS003Y (Millipore) water purification system.

\section{Sample preparation}

Samples with different and relatively low hCG levels are needed to validate the method. As such, two urine samples, assumed to have adequate hCG levels, have been collected and combined: one $\left(X_{0}\right)$ from an apparently healthy adult man, with urinary hCG concentrations assumed to be zero, and another $\left(X_{11}\right)$ from a pregnant woman at the fifth week of pregnancy (after informed consent), whose circulating hCG of $5110 \mathrm{mIU} / \mathrm{ml}$ urine typically reaches the expected levels of approximately $5000 \mathrm{mIU} / \mathrm{ml}$ urine. Dilutions of $X_{11}$ have been made with $X_{0}$, in different proportions, to obtain a sufficient number of samples within the range of low and very low hCG concentrations (Table 1). An equivalent procedure was used in connectin with quality control samples (designated as primary control samples), which were diluted with purified de-ionized water to obtain secondary control samples. These samples represented a range from approximately $1-500 \mathrm{mIU} / \mathrm{ml}$. From all urine and control samples, an adequate number of replicates were prepared by depositing sample aliquots of at least $0.5 \mathrm{ml}$ into $4 \mathrm{ml}$ polyethylene tubes, which were kept frozen until analysis. Replicates of a blank sample $\left(X_{\mathrm{B}}\right)$ have been prepared from hCG sample diluent's.

\section{Assay validation}

Validation of immunoassays includes all of the procedures that demonstrate that a particular method used for quantitative measurement of analytes in a given biological matrix is reliable and reproducible for the intended use. The fundamental parameters for this validation include precision, accuracy, selectivity, sensitivity, and stability (FDA (Food and Drug Administration), 2001). The extent of the immunoassay validation procedure depends on the intended application of the method (Findlay et al., 2000) and on the assay's development and implementation level. For example, full validation is needed when developing and implementing a bioanalytical method for the first time. However, when changes are made to a previously validated method, different levels of partial validation may be defined which can range from as simple as one intra-assay accuracy and precision determination to a nearly full validation (FDA (Food and Drug Administration), 2001). Typical bioanalytical method changes that fall into this category include change in relevant concentration range, as is the case of the present study. Therefore, validation of the IMMULITE 2000 immunoassay for the quantification of very low levels of urinary hCG will address the following basic performance characteristics: precision, sensitivity, accuracy, and analyte stability. Certain secondary assay parameters (e.g., lower limit of detection and functional sensitivity) which can be derived from the basic characteristics will also be determined.

\section{Precision}

Precision describes the closeness of agreement between a series of measurements obtained from multiple sampling of the same homogeneous sample under the prescribed conditions (FDA (Food and Drug Administration), 2001). For the precision related performance characteristics of the method, intra- or within-precision (repeatability) and interassay precision (reproducibility) are usually considered. 
Table 1. Intra- and inter-assay precision for the urine samples prepared to validate an immunometric assay for the quantification of low levels of hCG in urine

\begin{tabular}{|c|c|c|c|c|c|}
\hline Sample ID & Run 1 & Run 2 & Run 3 & Mean \pm SD $^{\mathrm{a}}$ & $C V \%{ }_{\text {Inter }}^{b}$ \\
\hline \multicolumn{6}{|l|}{$X_{1}$} \\
\hline Mean ${ }^{c}$ & 7.66 & 8.55 & 8.00 & \multirow[t]{3}{*}{$8.07 \pm 0.447$} & \multirow[t]{3}{*}{5.53} \\
\hline $\mathrm{SD}^{\mathrm{d}}$ & 0.253 & 0.370 & 0.464 & & \\
\hline $\mathrm{CV} \%{ }_{\text {Intra }}^{\mathrm{e}}$ & 3.30 & 4.36 & 5.80 & & \\
\hline \multicolumn{6}{|l|}{$X_{2}$} \\
\hline Mean & 14.55 & 15.73 & 14.5 & \multirow[t]{3}{*}{$14.9 \pm 0.709$} & \multirow[t]{3}{*}{4.75} \\
\hline $\mathrm{SD}$ & 0.477 & 0.48 & 0.374 & & \\
\hline CV\% Intra & 3.28 & 3.03 & 2.58 & & \\
\hline \multicolumn{6}{|l|}{$X_{3}$} \\
\hline Mean & 27.08 & 29.1 & 27.0 & \multirow[t]{3}{*}{$27.7 \pm 1.21$} & \multirow[t]{3}{*}{4.37} \\
\hline $\mathrm{SD}$ & 0.819 & 0.79 & 0.672 & & \\
\hline CV\% Intra & 3.02 & 2.72 & 2.49 & & \\
\hline \multicolumn{6}{|l|}{$X_{4}$} \\
\hline Mean & 48.2 & 52.3 & 47.7 & \multirow[t]{3}{*}{$49.4 \pm 2.51$} & \multirow[t]{3}{*}{5.07} \\
\hline SD & 1.55 & 1.77 & 1.48 & & \\
\hline CV\% $\%_{\text {Intra }}$ & 3.22 & 3.39 & 3.09 & & \\
\hline \multicolumn{6}{|l|}{$X_{5}$} \\
\hline Mean & 100 & 98.1 & 90.6 & \multirow[t]{3}{*}{$96.2 \pm 4.98$} & \multirow[t]{3}{*}{5.17} \\
\hline $\mathrm{SD}$ & 2.39 & 4.30 & 1.94 & & \\
\hline CV\% Intra & 2.39 & 4.40 & 2.14 & & \\
\hline \multicolumn{6}{|l|}{$X_{6}$} \\
\hline Mean & 167 & 159 & 146 & \multirow[t]{3}{*}{$157 \pm 10.7$} & \multirow[t]{3}{*}{6.82} \\
\hline $\mathrm{SD}$ & 2.55 & 4.64 & 3.66 & & \\
\hline CV\% ${ }_{\text {Intra }}$ & 1.53 & 2.91 & 2.52 & & \\
\hline \multicolumn{6}{|l|}{$X_{7}$} \\
\hline Mean & 298 & 286 & 274 & \multirow[t]{3}{*}{$286 \pm 11.7$} & \multirow[t]{3}{*}{4.09} \\
\hline $\mathrm{SD}$ & 7.23 & 11.7 & 12.1 & & \\
\hline CV\% Intra & 2.43 & 4.09 & 4.40 & & \\
\hline \multicolumn{6}{|l|}{$X_{11}$} \\
\hline Mean & 5178 & 4670 & 4561 & \multirow[t]{3}{*}{$4803 \pm 329$} & \multirow[t]{3}{*}{6.85} \\
\hline $\mathrm{SD}$ & 184 & 139 & 103 & & \\
\hline CV\% Intra & 3.55 & 2.97 & 2.27 & & \\
\hline
\end{tabular}

${ }^{a}$ Mean concentration and standard deviation of the assayed samples over three different runs.

${ }^{\mathrm{b}}$ Inter-assay imprecision, calculated as the variation coefficient of the mean concentrations of the assayed samples over the three different runs.

${ }^{\mathrm{c}}$ Mean value, expressed as $\mathrm{mIU} \mathrm{hCG} / \mathrm{ml}$ urine, for $n=10$ replicates from each of the $X_{i}$ samples assayed on the same day.

${ }^{\mathrm{d}}$ Standard deviation, expressed as $\mathrm{mIU}$ hCG $/ \mathrm{ml}$ urine, for $n=10$ replicates from each of the $X_{i}$ samples assayed on the same day.

${ }^{\mathrm{e}}$ Intra- or within-assay imprecision, calculated as the variation coefficient of assayed replicates from the corresponding sample.

The precision at each concentration level can be measured as the standard deviation (SD) or percentage of variation variation coefficient $(\mathrm{CV} \%)$ - around the mean value of assayed replicates (Findlay et al., 2000).

Prepared urine samples were assayed in replicate $(n=10)$, three times over the course of 5 days, one run per day, for a total of 3 runs and 30 replicates for each hCG concentration level. Intra- or within-assay imprecision for each of the different levels of hCG concentration was measured as the variation coefficient $(\mathrm{CV} \%)_{\text {Intra }}$ on the same run for each of the samples. Inter-assay imprecision was calculated as the variation coefficient $(\mathrm{CV} \%)_{\text {Inter }}$ for each sample and for the three runs performed.

\section{Sensitivity}

Sensitivity is "the resolving power at zero dose" (Ekins and Edwards, 1997). This means that maximal analytical sensitivity is achieved when the imprecision of the zero dose measurement is least, on the grounds that this quantity represents the most appropriate numerical indicator of a measuring system's ability to respond to a small amount or weak signal. This statistic essentially determines the system's detection limit (Ekins and Edwards, 1997).

One of the fundamental characteristics of any bioanalytical method is the smallest concentration that can be reliably measured. By definition $\left(\right.$ DPC $^{\circledR}$ (Diagnostic 
Products Corporation), 2001; FDA (Food and Drug Administration), 2001; Findlay et al., 2000), the assay's limit of detection (LOD) is "the lowest concentration that can be distinguished from background noise", i.e., the minimum detectable concentration of the analyte. It can be calculated as the concentration corresponding to the signal obtained at two standard deviations from the mean signal of blank sample replicates (Ersfeld et al., 2004) according to Eq. (1)

$$
\mathrm{LOD}=\bar{X}_{\mathrm{B}}+k * \mathrm{SD}_{\mathrm{B}},
$$

where $\bar{X}_{\mathrm{B}}$ is the mean signal of blank sample replicates, prepared independently and assayed sequentially under the same conditions, and $\mathrm{SD}_{\mathrm{B}}$ the standard deviation associated with the $X_{\mathrm{B}}$ replicate results. Assuming that these results have a normal distribution, the factor $k$ is made equal to 2 , for a confidence level of $95 \%$. In the present study, forty replicates of the blank sample were assayed and used to determine $\bar{X}_{\mathrm{B}}$ and $\mathrm{SD}_{\mathrm{B}}$.

If, as is the case, a regression line of "standard deviation versus analyte concentration" is available, LOD can also be calculated directly as $k$ times the intercept $\left(\mathrm{SD}_{0}\right)$ of this line (Ekins and Edwards, 1997). Knowledge of the assay precision profile (defined as the graphical representation of the relationship between the variation coefficient in analytical measurements of a sample under specified conditions and the nominal concentration of the analyte in that sample) yielded by each of two different methods or analytical techniques immediately reveals which one yields a lower value of $\mathrm{SD}_{0}$ and hence which is more "sensitive" (Ekins and Edwards, 1997).

Prepared urine samples were assayed for a total of 30 replicates for each hCG concentration level. The assay's detection limit or analytical sensitivity was determined from a least squares regression analysis of standard deviation versus hCG concentration for each sample tested.

In the "real world", analytical sensitivity has limited practical value, since at this level of concentrations, and even at concentrations significantly above it, imprecision can be so great that results do not reproduce in terms of being of real analytical utility $\left(\mathrm{DPC}^{\circledR}\right.$ (Diagnostic Products Corporation), 2001). The concept of limit of quantification (LOQ), defined as the concentration corresponding to the blank signal plus 10 times the standard deviation of the blank, and which can be calculated according to

$\mathrm{LOQ}=\bar{X}_{\mathrm{B}}+10 \times \mathrm{SD}_{\mathrm{B}}$

has been used to represent the lowest measurable concentration that is analytically useful. Based on the line of "standard deviation versus analyte concentration", LOQ can also be calculated by multiplying by 10 the intercept $\left(\mathrm{SD}_{0}\right)$ of this line.
Some years ago, another concept, termed "functional sensitivity" and defined as the "lowest concentration at which an assay can report clinically useful results", was developed to provide laboratories with an objective and clinically meaningful indication of the practical lower limit of the assay (Spencer, 1989). Functional sensitivity is commonly determined as the mean analyte concentration at which the mean inter-assay imprecision, expressed as CV\%, is $<20 \%$ (Ersfeld et al., 2004). However, for some assays, a $\mathrm{CV} \%$ greater (lesser) than $20 \%$ is used since it is consistent with clinically reliable and informative results (DPC ${ }^{\circledR}$ (Diagnostic Products Corporation), 2006).

Prepared urine samples (30 replicates for each hCG concentration level) were assayed to assess the day-today precision and the $\mathrm{CV} \%$ was calculated for each sample tested. The functional sensitivity was determined as the mean hCG concentration at which the mean imprecision, expressed as $\mathrm{CV} \%$, reached $15 \%$.

\section{Accuracy}

Accuracy is a concept that expresses the closeness of agreement between a measured test value and its nominal or known true value (FDA (Food and Drug Administration), 2001; Findlay et al., 2000). As a basic performance characteristic of a bioanalytical method, accuracy describes the closeness of agreement between a mean test results obtained by the method and the nominal or known true value (concentration) of the analyte (FDA (Food and Drug Administration), 2001). Accuracy is determined by replicate analysis of samples containing known amounts of the analyte. It is recommended that a minimum of three concentrations in the range of expected concentrations and a minimum of five determinations per concentration should be used (FDA (Food and Drug Administration), 2001). For the validation of IMMULITE 2000 immunoassay accuracy, 11 urine samples and 8 control samples (consisting of 3 original samples and 5 obtained by dilution) were assayed in replicate $(n=30)$. Closeness of mean hCG results obtained by each of these samples in relation to the actual (or expected) concentration, analysed by least squares linear regression, was taken as accuracy of the method for each of the assayed hCG concentrations included in the studied concentration range.

\section{Analyte stability}

The stability of an analyte, in a particular biological fluid, is a function of storage conditions, analyte chemical properties, matrix, and container system (FDA (Food and Drug Administration), 2001). In the case of the hCG assay, instability of the analyte can be a potential problem. For assay validation, stability 
procedures should then evaluate hCG stability during sample handling, after short- and long-term storage, and after going through freeze and thaw cycles, based on the expected real conditions that samples will be subject to during the whole analytical process.

In the present work, hCG stability was considered only in relation to storage (time and temperature) since real samples are frozen immediately after collection and consequently sample handling will not be a significant problem. Stability of hCG was therefore assessed from results of the studies where replicates from the urine samples were analysed for different storage times and conditions. For long-term storage, one set of replicates for each concentration level was assayed and kept frozen until re-assaying more than 3 months later (the expected storage period under real conditions of routine testing). For short-term stability testing, results used were those from two sets of samples prepared at the same time, defrosted before analysis within a five days difference period, and from one set of samples assayed after thawing at room temperature and re-assayed at days 3 and 5, being kept at $2-8^{\circ} \mathrm{C}$ between runs.

\section{Results and discussion}

The assay was always performed strictly according to the manufacturer's instructions $\left(\right.$ DPC $^{\circledR}$ (Diagnostic Products Corporation), 2005) and after all the routine maintenance procedures as defined in the IMMULITE 2000 operator's manual. Results were expressed as mIU $\mathrm{hCG} / \mathrm{ml}$ urine.

\section{Precision}

Table 1 shows results for the determination of withinand inter-assay precision. The determinations were performed at concentrations expected to be encountered mainly during the first part of the gestational period (3-4 weeks, according to limits reported as guidelines by IMMULITE 2000 manufacturer's studies) (DPC ${ }^{\circledR}$ (Diagnostic Products Corporation), 2005). Sample $X_{1}$ (characterized by a mean of $8.07 \mathrm{mIU} / \mathrm{ml}$ and a standard deviation in reproduction analysis of $0.45 \mathrm{mIU} / \mathrm{ml}$ ) is very close to the non-pregnant usual limit $(5 \mathrm{mIU} / \mathrm{ml})$, presenting a $\mathrm{CV} \%$ Inter of $5.5 \%$ and a variation coefficient for repetition analysis which ranges from $3.3 \%$ to $5.8 \%$. The other samples, with higher hCG concentrations, corresponding to gestational age from conception of up to 3-4 weeks, or equivalently to 5-6 weeks since last menstrual period, present very similar values for $\mathrm{CV} \%_{\text {Intra }}$ and for $\mathrm{CV} \%_{\text {Inter }}$, pointing to a good performance in the precision of the IMMULITE hCG assay for urine within the critical range from around $5-5000 \mathrm{mIU} / \mathrm{ml}$. This means that, under normal circumstances, an early pregnancy loss, identified by its typical "rise and fall" pattern of urinary hCG, can be quite detectable by the immunoassay, even at very low gestational ages, providing that the difference between hormonal elevation and fall is within the intra-assay precision for the hCG concentration level. Inter-assay precision was less than $15 \%$, ranging from approximately 4-7\%. Similar precision studies in serum samples conducted by the IMMULITE 2000 manufacturer and reported in the assay package insert $\left(\mathrm{DPC}^{\circledR}\right.$ (Diagnostic Products Corporation), 2005) have shown comparable precision parameters with $\mathrm{CV} \%_{\text {Intra }}$ and $\mathrm{CV} \%_{\text {Inter }}$ ranging from $2.5 \%$ to $6.6 \%$ and $4.8 \%$ to $7.4 \%$, respectively.

\section{Sensitivity}

Minimum detectable concentration (LOD) and minimum measurable concentration (LOQ) were determined as the concentrations corresponding to the signals obtained at two and at 10 standard deviations from the mean of the signal of the blank (zero-concentration) sample, respectively. Forty replicates were assayed and used to determine the mean and standard deviation and to extrapolate apparent concentrations at two $\left(\mathrm{LOD}_{\mathrm{B}}\right)$ and at $10\left(\mathrm{LOQ}_{\mathrm{B}}\right)$ standard deviations from this mean. $\mathrm{LOD}_{\mathrm{B}}$ and $\mathrm{LOQ}_{\mathrm{B}}$ were found to be 1.2 and $2.9 \mathrm{mIU} / \mathrm{ml}$, respectively. The equivalent basic performance parameters were calculated by using the intercept of the regression line of standard deviation versus analyte concentration $\left(\mathrm{SD}_{0}\right)$ as the estimate of the standard deviation for the hCG zero concentration (Fig. 1). The $\mathrm{LOD}_{0}$ and $\mathrm{LOQ}_{0}$ values determined in this way were 1.99 and $9.9 \mathrm{mIU} / \mathrm{ml}$, respectively. Therefore, assuming $\operatorname{LOD}_{\mathrm{B}}$ as the lowest concentration that can be distinguished from background noise, the assay's limit of detection will be comparable to the one reported for serum $(1.1 \mathrm{mIU} / \mathrm{ml})$ by the IMMULITE 2000 assay product insert (DPC ${ }^{\circledR}$ (Diagnostic Products Corporation), 2005).

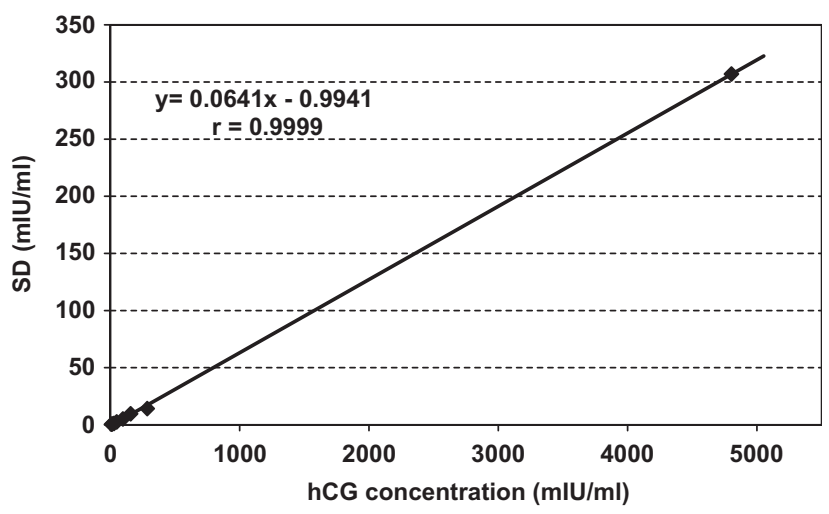

Fig. 1. Variation of standard deviation with observed hCG concentration in urine samples. 
Considering either $\mathrm{LOD}_{\mathrm{B}}$ or $\mathrm{LOD}_{0}$, it is evident that the minimum detectable concentration of the assay was less than the non-pregnant usual concentration $(5 \mathrm{mIU} / \mathrm{ml})$, which is relevant for the objectives of the immunoassay's application. The lowest measurable concentration for the IMMULITE 2000 assay $\left(\mathrm{LOQ}_{\mathrm{B}}=2.9 \mathrm{mIU} / \mathrm{ml}\right)$ was also less than the non-pregnant confident limit considered by Chard (1992) and other authors. Considering the limit of $15 \%$ or even $10 \%$ of $\mathrm{CV} \%$ Inter for the determination of the assay's functional sensitivity (see Table 1), it is also evident that the lowest concentration at which the IMMULITE 2000 immunoassay for urinary hCG can report useful results is included in the range of concentrations that have been studied in the present work, meaning that the IMMULITE 2000 immunoassay is able to measure and report urinary hCG concentrations around and above $5 \mathrm{mIU} / \mathrm{ml}$. From these findings, it can be concluded that the IMMULITE 2000 hCG immunoassay by $\mathrm{DPC}^{\circledR}$ can identify, with relatively high confidence, non-pregnant women and the typical "rise and fall" pattern of early pregnancy loss through analysis of urine samples.

\section{Accuracy}

For the determination of immunoassay accuracy, urine and control samples were assayed under several dilutions (Table 2): a urine sample from a pregnant woman was diluted with urine from an apparently healthy adult man, and three control samples were diluted with deionised water, until measurable results were obtained. Resulting samples were assayed in replicate $(n=30)$ and closeness of mean hCG results in relation to expected concentrations was analysed by least squares linear regression (Fig. 2). Equation from regression analysis (observed $=0.99$ expected +8.55 , with a correlation coefficient of 0.993 ) demonstrates very good immunoassay accuracy for the studied range of hCG concentrations. Since controls are human serum-based samples comprising hCG concentrations from around 5 to almost $500 \mathrm{mIU} / \mathrm{ml}$, the good performance of the linear relationship between expected and observed hCG concentrations for urine and control samples altogether is indicative that the assay is not affected by the sample matrix.

\section{Analyte stability}

Concerning analyte stability, several studies have shown that urinary hCG was stable under a variety of conditions including storage at $4{ }^{\circ} \mathrm{C}$ (refrigerated samples) and $-20^{\circ} \mathrm{C}$ (frozen samples) with or without preservatives (Alfthan et al., 1992; Ajubi et al., 2005; DPC ${ }^{\circledR}$ (Diagnostic Products Corporation), 2001).

Table 2. Expected or reported and observed hCG concentration in urine and control samples.

\begin{tabular}{|c|c|c|c|c|}
\hline \multirow[t]{2}{*}{ Sample } & \multirow[t]{2}{*}{ Dilution $\left(X_{0}: X_{11}\right)$} & \multicolumn{3}{|c|}{ hCG concentration $(\mathrm{mIU} / \mathrm{ml})$} \\
\hline & & Expected & Reported $^{\text {a }}$ Mean \pm SD; Range & Observed Mean \pm SD \\
\hline \multicolumn{5}{|l|}{ Urine samples } \\
\hline$X_{1}$ & $1: 800$ & 6.4 & & $8.07 \pm 0.45$ \\
\hline$X_{2}$ & $1: 400$ & 12.8 & & $14.9 \pm 0.71$ \\
\hline$X_{3}$ & $1: 200$ & 25.6 & & $27.7 \pm 1.2$ \\
\hline$X_{4}$ & $1: 100$ & 51.1 & & $49.4 \pm 2.5$ \\
\hline$X_{5}$ & $1: 50$ & 102 & & $96.2 \pm 5.0$ \\
\hline$X_{6}$ & $1: 30$ & 170 & & $157 \pm 11$ \\
\hline$X_{7}$ & $1: 15$ & 341 & & $286 \pm 12$ \\
\hline$X_{8}$ & $1: 8$ & 639 & & $582 \pm 31$ \\
\hline$X_{9}$ & $1: 5$ & 1022 & & $995 \pm 20$ \\
\hline$X_{10}$ & $1: 2$ & 2555 & & $3087 \pm 57$ \\
\hline$X_{11}$ & $0: 1$ & $5110^{\mathrm{b}}$ & & $4803 \pm 329$ \\
\hline Quality control samples & Dilution $^{\mathrm{c}}$ & & & \\
\hline$C_{1}$ & - & 11.1 & $11.1 \pm 1.01 ;(9.1 ; 13.1)$ & $10.4 \pm 0.29$ \\
\hline$C_{1,2}$ & $1: 2$ & 5.5 & & $5.03 \pm 0.17$ \\
\hline$C_{2}$ & - & 44 & $44 \pm 3.0 ;(38 ; 50)$ & $40.5 \pm 0.3$ \\
\hline$C_{2,2}$ & $1: 2$ & 22 & & $19.9 \pm 0.4$ \\
\hline$C_{2,10}$ & $1: 10$ & 4.4 & & $3.74 \pm 0.12$ \\
\hline$C_{3}$ & - & 430 & $430 \pm 25 ;(380 ; 480)$ & $415 \pm 8$ \\
\hline$C_{3,2}$ & $1: 2$ & 215 & & $210 \pm 4$ \\
\hline$C_{3,10}$ & $1: 10$ & 43 & & $37.5 \pm 1.1$ \\
\hline
\end{tabular}

\footnotetext{
${ }^{\mathrm{a}}$ From Diagnostic Products Corporation-DPC ${ }^{\circledR}$, Multivalent Control Module (IMMULITE data sheet).

${ }^{b}$ Results calculated as average of mean values from three different assays of 10 replicates.

${ }^{\mathrm{c}}$ Dilution with de-ionized water.
} 


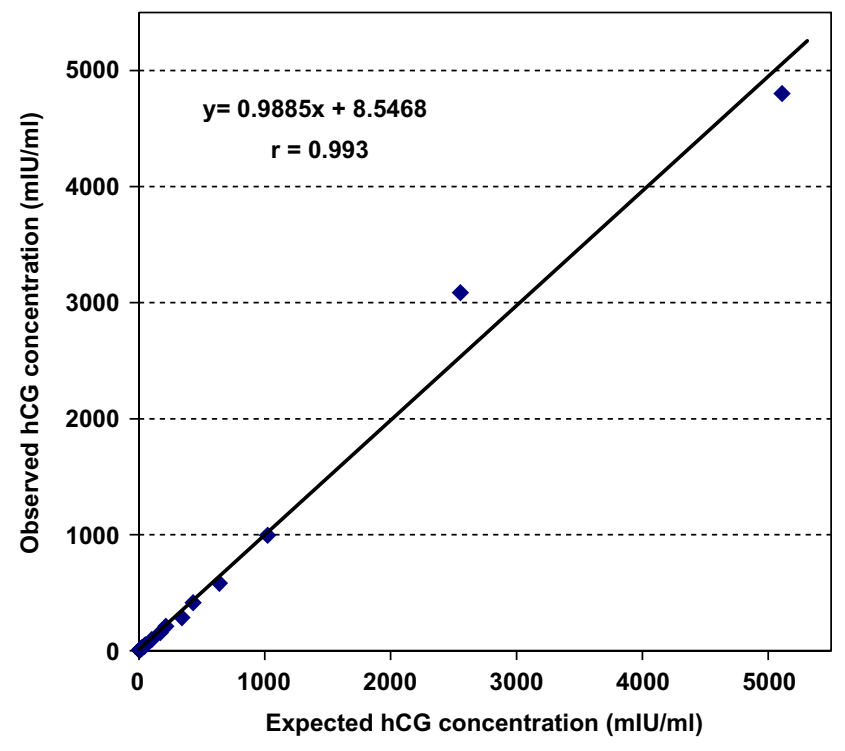

Fig. 2. Correlation between expected and observed hCG concentrations in urine samples.

However, findings from these studies do not agree in relation to the duration of the stability period. In the present study, analyte stability was assessed by using all prepared urine samples in two separate studies, in order to determine long- and short-term stability for either frozen samples, or refrigerated and frozen samples, respectively.

Student's $t$-test for paired samples used in the longterm stability study showed that results from the same set of samples assayed and kept frozen until re-assaying more than 3 months later were not statistically significant $(p>0.05)$. Similarly, a short-term stability study involving two sets of frozen samples prepared at the same time and assayed within a 5-day period showed, through a Student's $t$-test for independent samples, that, as expected, differences are not statistically significant $(p>0.05)$. However, when the same set of samples was assayed after thawing at room temperature and re-assayed at days 3 and 5 , being kept at $2-8{ }^{\circ} \mathrm{C}$ between runs, different results were obtained: paired samples $t$-test for results from the assay at day 1 and the re-assay at day 3 showed that differences are not statistically significant; on the other hand, differences are significant $(p<0.001)$ between results from the assay at day 1 and the re-assay at day 5, or at day 3 and the reassay at day 5. Findings from these studies led to the conclusion that urine samples can be considered stable for up to $48 \mathrm{~h}$ if maintained at temperatures of $2-8{ }^{\circ} \mathrm{C}$ (refrigerated) and for at least 3 months at temperatures around $-20{ }^{\circ} \mathrm{C}$. The results indicate that the recommended (DPC ${ }^{\circledR}$ (Diagnostic Products Corporation), 2001) storage conditions (refrigerated for $48 \mathrm{~h}$ or frozen at $-20^{\circ} \mathrm{C}$ for longer pre-analysis periods) are adequate.

\section{Acknowledgments}

The authors of this paper thank AMERLAB, the IMMULITE 2000 manufacturer's representative in Portugal, for permitting the use of its IMMULITE 2000 analyzer and for the helpful assistance not only with the adjustments in the software reporting range, but also with all the work performed on urine samples for the hCG immunoassay validation and in real samples routine testing.

\section{References}

Ajubi, N.E., Nijholt, N., Wolthuis, A., 2005. Quantitative automated human chorionic gonadotropin measurement in urine using the modular analytics E170 module (Roche). Clin. Chem. Lab. Med. 43 (1), 68-70.

Alfthan, H., Haglund, C., Dabek, J., Stenman, U.-H., 1992. Concentrations of human choriogonadotropin, its $\beta$ subunit, and the core fragment of the $\beta$-subunit in serum and urine of men and nonpregnant women. Clin. Chem. 38 (10), 1981-1987.

Bhatt, R.V., 2000. Environmental influence on reproductive health. Int. J. Gynecol. Obstetr. 70, 69-75.

BDS (BioDetection Systems B.V.), 2003. In house validation protocol for the analysis of dioxins and dioxin-like compounds using the DR-CALUX ${ }^{\mathbb{R}}$ bioassay: extraction, clean-up and DR-CALUX ${ }^{\circledR}$ bioanalysis.

Buck, G.M., Lynch, C.D., Stanford, J.B., Sweeney, A.M., Schieve, L.A., Rockett, J.C., Selevan, S.G., Schrader, S.M., 2004. Prospective pregnancy study designs for assessing reproductive and developmental toxicants. Environ. Health Perspect. 112 (1), 79-86.

Chapin, R.E., Robbins, W.A., Schieve, L.A., Sweeney, A.M., Tabacova, S.A., Tomashek, K.M., 2004. Off to a good start: the influence of pre- and periconceptional exposures, parental fertility, and nutrition on children's health. Environ. Health Perspect. 112 (1), 69-78.

Chard, T., 1992. Pregnancy tests: a review. Hum. Reprod. 7 (5), 701-710.

Cole, L.A., 1997. Immunoassay of human chorionic gonadotropin, its free subunits, and metabolites. Clin. Chem. 43 (12), 2233-2243.

Cole, L.A., Shahabi, S., Butler, S.A., Mitchell, H., Newlands, E.S., et al., 2001. Utility of commonly used commercial human chorionic gonadotropin immunoassays in the diagnosis and management of trophoblastic diseases. Clin. Chem. 47 (2), 308-315.

DPC $^{\circledR}$ (Diagnostic Products Corporation), 2001. Analytical sensitivity, functional sensitivity:how low can you go? <http://www.dpcweb.com/documents/news\&views/ spring01/performancemeasures.html $\rangle$.

DPC $^{\circledR}$ (Diagnostic Products Corporation), 2005. IMMULITE 2000 HCG. PIL2KCG-19.

DPC $^{\circledR} \quad$ (Diagnostic Products Corporation), 2006. CON6 multivalent control module (IMMULITE $\AA$ data sheet). PICON6I-16.

Ekins, R., Edwards, P., 1997. On the meaning of "sensitivity". Clin. Chem. 43 (10), 1824-1837. 
Ersfeld, D.L., Rao, D.S., Body, J.J., Sackrison, J.L., Miller, A.B.etal., 2004. Analytical and clinical validation of the 25 $\mathrm{OH}$ vitamin $\mathrm{D}$ assay for the LIAISON $^{\circledR}$ automated analyzer. Clin. Biochem. 37 (10), 867-874.

Findlay, J.W.A., Smith, W.C., Lee, J.W., Nordblom, G.D., Das, I., et al., 2000. Validation of immunoassays for bioanalysis: a pharmaceutical industry perspective. J. Pharm. Biomed. Anal. 21, 1249-1273.

FDA (Food and Drug Administration), 2001. Guidance for Industry: Bioanalytical method validation. US Department of Health and Human Services.

NCCLS (National Committee for Clinical Laboratory Standards), 1986. Evaluation of the linearity of quantitative analytical methods. NCCLS document EP-6P, NCCLS, Wayne.

NCCLS (National Committee for Clinical Laboratory Standards), 1999. Evaluation of precision performance of clinical chemistry devices; approved guideline. NCCLS Document EP-5A, NCCLS, Wayne.

Reis, M.F., Machado, A.A.S.C., Abdulla, M., Canário, J., Silva, J.C., 1994. Validation of the cumulative or replicate NAA method for the determination of trace elements in biological materials. Biol. Trace Elem. Res. 43/45, 585-596.

Sackrison, J.L., Ersfeld, D.L., Miller, A.B., Olson, G.T., MacFarlane, G.D., 2002. Development of a sensitive, automated, non-extracted, direct LIAISON ${ }^{\circledR}$ immunoassay for $25 \mathrm{OH}$ vitamin D. Clin. Chem. 48, A122.

Shah, V.P., Midha, K.K., Findlay, J.W.A., Hill, H.M., Hulse, J.D., et al., 2000. Bioanalytical method validation-a revisit with a decade of progress. Pharm. Res. 17 (12), 1551-1557.

Shahara, F.I., Seifer, D.B., Flaws, J.A., 1998. Environmental toxicants and female reproduction. Fertil. Steril. 70 (4), 613-622.

Spencer, C.A., 1989. Thyroid profiling for the 1990s: free T4 estimate or sensitive TSH measurement. J. Clin. Immunoassay $12,82-89$.

Tingen, C., Stanford, J.B., Dunson, D.B., 2004. Methodologic and statistical approaches to studying human fertility and environmental exposure. Environ. Health Perspect. 112 (1), 87-93.

Trullols, E., Ruisánchez, I., Rius, F.X., Huguet, J., 2005. Validation of qualitative methods of analysis that use control samples. Trends Anal. Chem. 24 (6), 516-524.

Vankrieken, L., Sibley, P., Kelly, J., 2000. HCG and subunits: DPC assay specificities and clinical utility in obstetrical care and oncology. Technical Report, DPC ${ }^{\circledR}$.

Wilcox, J.F., Weinberg, C.R., O'Connor, J.F., Braid, D.D., Schlatterer, J.P., et al., 1988. Incidence of early loss of pregnancy. N. Engl. J. Med. 319 (4), 189-194. 